\title{
Fare storia: il laboratorio didattico sull'eccidio di La Bettola
}

L'articolo illustra lo svolgimento di un laboratorio didattico per due classi terze di scuola secondaria di $I^{\circ}$ grado che ricostruisce l'eccidio nazista del giugno 1944 a La Bettola $(R E)$. L'analisi e l'interpretazione delle differenti fonti hanno consentito di fare storia, rendendo i ragazzi protagonisti nella costruzione del loro apprendimento e attivando molteplici competenze cognitive e trasversali.

This article focuses on the educational workshop for the junior secondary school, organized in La Bettola in order to approach the Nazi massacre of June 1944. Using different sources, the students became protagonists in the construction of their learning and activating multiple cognitive skills.

\section{Lo scopo}

In occasione del $70^{\circ}$ anniversario della Liberazione, per le due classi terze della scuola secondaria di primo grado San Vincenzo de' Paoli (RE), è stato avviato un laboratorio di indagine storica basato sull'analisi delle fonti riguardanti l'eccidio del 23 giugno del 1944 a La Bettola. Il progetto si inseriva all'interno delle attività promosse dall'Officina didattica della storia: un'iniziativa patrocinata dall'Istituto per la storia della Resistenza e della società contemporanea in provincia di Reggio Emilia (Istoreco) con lo scopo di avvicinare gli studenti allo studio e all'approfondimento di eventi legati alla storia contemporanea locale. Nella convinzione che imparare e insegnare la storia non consistano unicamente 
nel trasmettere conoscenze, ma siano anche un "fare", si è deciso di valorizzare la centralità dell'apprendimento attraverso un percorso laboratoriale. Pur consapevoli della complessità organizzativa e logistica che un progetto di questo tipo richiede, si è privilegiata una didattica in grado di coinvolgere attivamente e in una modalità paritaria di lavoro e di cooperazione studenti e docenti per la costruzione del sapere.

\section{La scelta del tema}

Affinché l'esperienza risultasse significativa, si è proposto un episodio leggibile sul territorio. L'oggetto d'indagine è stato quindi individuato nella strage de La Bettola, una località della collina reggiana, anche perché i destini di due protagoniste di quella tragica vicenda si incrociano alla storia della scuola: una delle vittime, l'allora ventiduenne Wilma Varini, era stata allieva della San Vincenzo e, nel maggio del 1944, aveva conseguito il diploma magistrale; una sopravvissuta, Liliana Del Monte, all'epoca dei fatti undicenne, fu affidata all'Istituto che in quegli anni era un orfanotrofio.

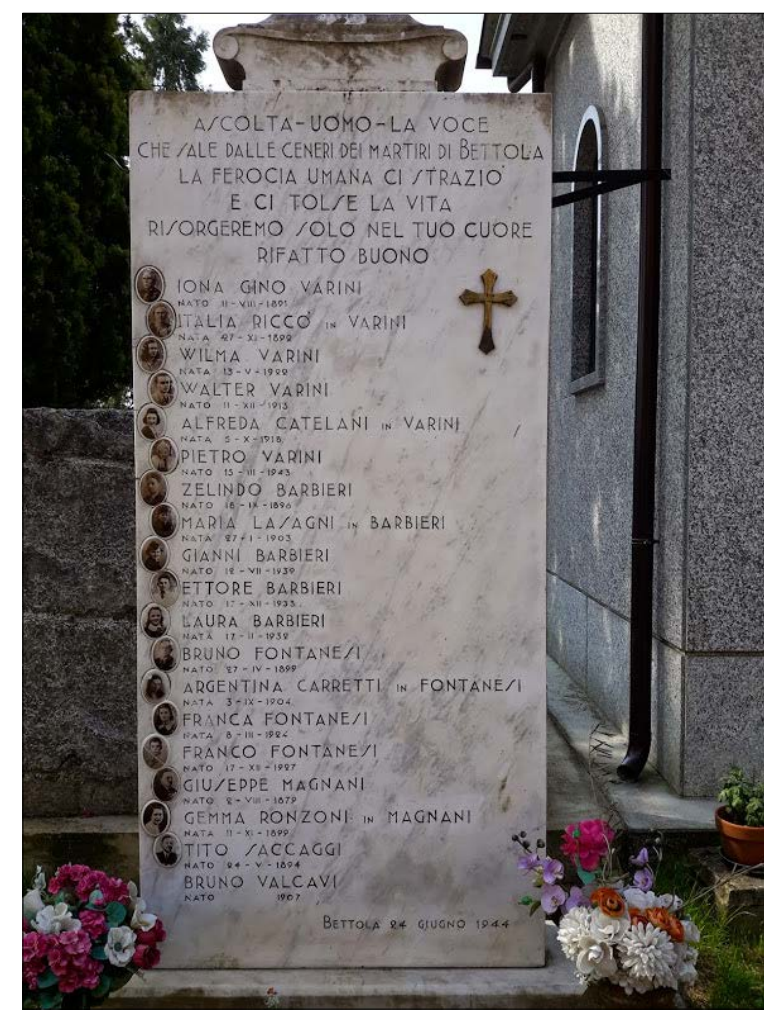

Foto del Cippo per le vittime reggiane presso il cimitero monumentale di Reggio Emilia

\section{Documenti, luoghi, persone, monumenti}

Punto di partenza è stata la lettura in classe del romanzo di Liliana Manfredi $\mathrm{Il}$ nazista e la bambina, la testimonianza letteraria della superstite che a 64 anni dai fatti ha ricostruito la sua drammatica vicenda: miracolosamente scampata alla 
furia omicida dei tedeschi durante un'azione di rappresaglia che costò la vita a 32 civili e a tre partigiani, gravemente ferita, venne poi salvata da un soldato addetto al giro di perlustrazione che ebbe compassione di lei e la portò sulla strada principale per essere soccorsa. Attraverso la lettura guidata del testo, i ragazzi sono stati sollecitati a cogliere i fatti propriamente storici rilevando persone, luoghi e tracce ancora visibili di quanto accaduto, come la localizzazione del teatro del massacro su Google Earth, i profili dei partigiani, dei carnefici e delle vittime con una ricerca in rete. Tra queste ultime gli studenti hanno individuato l'ex alunna Wilma Varini a cui è dedicato un commosso ricordo della madre superiora in occasione del trentennale della strage e un santino commemorativo dei membri della sua famiglia, documenti entrambi presenti nell'archivio scolastico. Nelle pagine finali del libro un'altra scoperta: la protagonista fino ai diciotto anni aveva trovato "riparo da altre violenze" [Manfredi 2008, 82] nei locali della scuola che allora era un orfanotrofio. Grazie a Wilma e a Liliana, non più solo nomi, ma persone, $\mathrm{i}$ ragazzi hanno così iniziato a percepire la vicinanza e la concretezza della vicenda ed erano motivati ad avviare l'approfondimento storico.

\section{I gruppi di lavoro}

Vista la mole di materiale da analizzare gli alunni delle due classi si sono suddivisi in base ai loro interessi e preferenze nei seguenti gruppi: fonti archivistiche, fonti iconografiche, fonti bibliografiche e fonti materiali.

Supervisionato da uno dei due docenti, il gruppo delle fonti archivistiche è stato accompagnato presso il Polo archivistico della città di Reggio dove ha potuto visionare la documentazione relativa alla strage. Lo storico Massimo Storchi e la responsabile della didattica di Istoreco Alessandra Fontanesi hanno illustrato ai ragazzi il contesto storico-geografico in cui maturò la tragedia e le dinamiche di quest'ultima. Gli alunni poi hanno potuto studiare i documenti più significativi quali ad esempio i risultati dell'indagine alleata effettuata nell'immediato dopoguerra e una recente video intervista al superstite Paolo Magnani.

I gruppi delle fonti iconografiche e materiali hanno ricercato in rete le immagini e i monumenti relativi all'eccidio e alla sua memoria nel territorio. In questo ambito è nato l'interesse di recarsi al cimitero monumentale con l'altro docente per osservare e fotografare il cippo dedicato alle vittime reggiane coinvolte nella strage perché sfollate in collina.

Gli alunni del gruppo delle fonti bibliografiche hanno selezionato nella biblioteca comunale i testi inerenti l'argomento e li hanno studiati e accuratamente sintetizzati. Dopo questa fase tutti i ragazzi si sono confrontati sui materiali e le 
informazioni raccolte arrivando a ricostruire un quadro complesso e dettagliato degli eventi del giugno 1944.

\section{L'incontro con la testimone}

Altro importantissimo momento è stato l'incontro e l'intervista a scuola con l'autrice, nonché sopravvissuta alla strage, Liliana Del Monte. Attraverso le domande dei ragazzi l'ottantenne testimone ha rievocato i momenti più tragici della notte di San Giovanni e delle ferite che quei fatti hanno lasciato nella sua vita, soffermandosi sugli anni trascorsi nell'ex orfanotrofio oggi sede della scuola. Dopo questo passaggio è maturata la consapevolezza di quanto sia importante nella ricerca storica il confronto, la critica e l'interpretazione delle molteplici fonti. Parallelamente si è cercato di far cogliere la differenza tra memorie individuali, spesso diverse e contrastanti tra loro, e l'elaborazione propriamente storica del passato.

\section{La visita sui luoghi della strage}

Durante gli incontri tra i docenti coinvolti nei progetti dell'Officina didattica della storia, è sembrato utile ampliare e condividere il nostro lavoro con altre scuole; è nata così una fruttuosa collaborazione con l'insegnante Elisa Maddalo, della classe IV della scuola primaria di Vezzano, del cui territorio comunale La Bettola fa parte, anch'ella interessata ad approfondire la storia contemporanea locale. In quest'ottica si è organizzata in primavera un'uscita sui luoghi della strage che ha permesso a tutti i discenti di rendersi protagonisti diretti dell'indagine storica. I bambini della scuola primaria hanno guidato, lungo il sentiero partigiano, i ragazzi di Reggio dalla località $\mathrm{Ca}$ ' di Rosino fino al ponte situato in prossimità de La Bettola. Una volta giunti a destinazione i più grandi, vicino all'attuale monumento, hanno loro raccontato i fatti lì avvenuti. Dal punto di vista della didattica è stato significativo per gli studenti della San Vincenzo preparare l'esposizione adattando il registro linguistico e i contenuti a destinatari così giovani. Il comune di Vezzano si è dimostrato particolarmente sensibile a questa iniziativa accogliendo poi nella sala consiliare tutti gli studenti in un momento di significativa commemorazione. Il Sindaco Mauro Bigi e l'Assessore all'istruzione Ilenia Rocchi hanno ringraziato $i$ partecipanti al progetto per aver studiato e mantenuto vivo il ricordo di eventi così vicini a noi, ma che sono a volte percepiti come molto lontani. L'occasione ha permesso di ampliare la documentazione delle fonti mate- 
riali perché l'assessore ha mostrato, letto e commentato ai ragazzi la prima lapide posta in ricordo delle vittime oggi conservata all'ingresso del Municipio.

Tutta la giornata è stata documentata dal regista Andrea Mainardi che, in collaborazione con Istoreco, ha poi realizzato il cortometraggio Sopra il ponte, un'accurata sintesi di tutto il progetto didattico.

\section{La mostra}

A coronamento dell'interessante indagine storica, in occasione del 25 aprile, i ragazzi hanno curato l'allestimento di una mostra aperta alla cittadinanza nei locali della scuola. Si sono occupati della progettazione, dell'allestimento degli spazi e della scelta dei materiali da esporre al pubblico. L'aspetto più complesso, ma anche più rilevante, è stata la redazione di quattro pannelli esplicativi intitolati: $I$ luoghi e l'azione partigiana, L'eccidio, Il dopo e La memoria. Per la loro realizzazione i ragazzi, sempre divisi in gruppi, hanno potenziato le competenze di scrittura applicando le procedure di ideazione, pianificazione, stesura e revisione del testo e scegliendo le immagini più adatte a corredo. L'intero progetto è stato ricostruito attraverso un cartellone didattico allo scopo di rendere espliciti i vari step del laboratorio: in alcune teche si sono sistemati libri e articoli scelti tra quelli delle fonti bibliografiche, mentre sono state allestite tre postazioni video dove era possibile visionare l'intervista a Paolo Magnani, l'incontro con Liliana

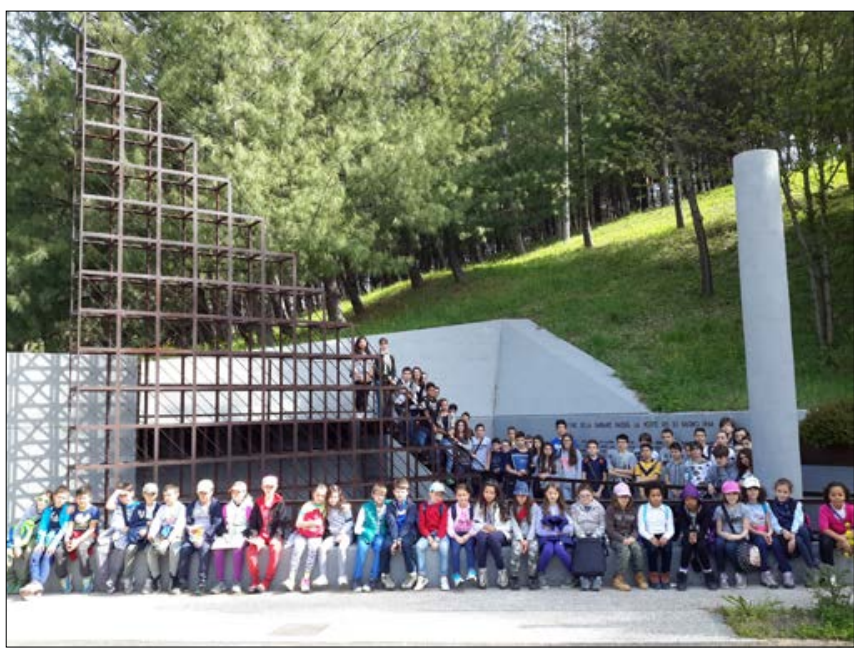

Foto di gruppo presso il monumento commemorativo a La Bettola

Manfredi e il corto

Sopra il Ponte.

Il giorno dell'inaugurazione, il 25 aprile 2015, i ragazzi hanno guidato i visitatori facendo loro da ciceroni. L'entusiasmo e la professionalità hanno caratterizzato questo alto momento formativo che li resi ancora una volta protagonisti attivi della costruzione del loro sapere attraverso la realizzazione di un compito autentico 
quale la mostra è stata.

\section{Conclusioni}

Il laboratorio è stato un ottimo strumento per potenziare le abilità proprie della disciplina e allo stesso tempo consolidare il senso di cittadinanza: rielaborare $\mathrm{i}$ fatti in forma orale e scritta per differenti usi; conoscere più approfonditamente il patrimonio storico dell'am-

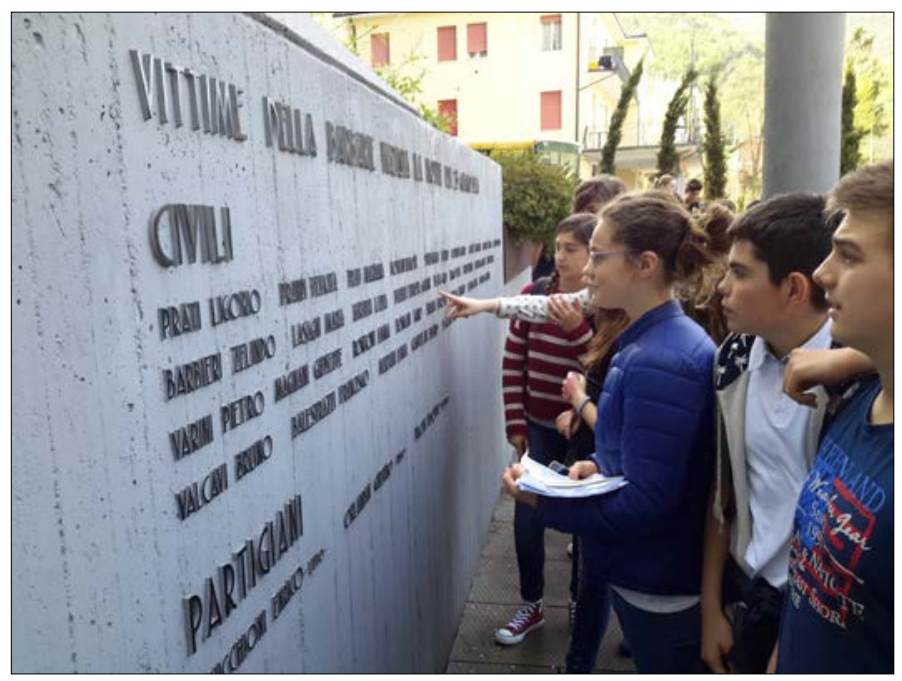

Dettaglio del monumento de La Bettola

biente circostante; acquisire e interpretare criticamente le informazioni raccolte attraverso diversi strumenti comunicativi, valutandone l'attendibilità e l'utilità, distinguendo fatti e opinioni; interagire in gruppo, comprendendo i diversi punti di vista, valorizzando le proprie e altrui capacità, gestendo la conflittualità, contribuendo all'apprendimento comune e alla realizzazione delle attività collettive; affrontare situazioni problematiche costruendo e verificando ipotesi, individuando le fonti e le risorse adeguate, raccogliendo e valutando i dati, proponendo soluzioni utilizzando contenuti e metodi diversi. 


\section{Bibliografia}

Casali M.N. 1999, Usi e rappresentazioni della memoria della strage di San Giovanni. Bettola, 23 giugno 1944, in Paggi L. (ed.) 1999, Le memorie della Repubblica, Scandicci: La Nuova Italia

De Prato T. 2014, “Rimasi solo in un silenzio agghiacciante”. La memoria di un testimone della strage della Bettola, "RS-Ricerche storiche", 118

Durchfeld M., Storchi M. 2014, La Bettola - La strage della notte di San Giovanni, Reggio Emilia: Istoreco, Comune di Vezzano sul Crostolo

Fangareggi S. 1981, L'eccidio di Bettola - Nel racconto di un sopravvissuto, "Reggio Storia", 4

Manfredi L. 2008, Il nazista e la bambina, Reggio Emilia: Aliberti

Storchi M. 2005, Anche contro donne e bambini - Le stragi tedesche e fasciste a Reggio Emilia, in Storchi M. (ed.) 2005, 20 mesi per la libertà: la guerra di liberazione dal Cusna al Po, Cavriago: Bertani \& C.

Vinceti R., 1945/1985, La Bettola, Vezzano sul Crostolo: Comune di Vezzano

\section{Risorse}

Istoreco

http://www.istoreco.re.it/default.asp?page $=460$,ITA

Anpi

http://www.anpireggioemilia.it/agenda-della-resistenza/1944-24-giugno-eccidio-dellabettola/

Monumento di La Bettola. Pdf

http://www.anpireggioemilia.it/wp-content/uploads/2015/03/Vezzano_ReportMonumenti_20140731.pdf

Comune di Vezzano per il $70^{\circ}$ della Resistenza

http://www.comune.vezzano-sul-crostolo.re.it/news/70\%C2\%B0-anniversario-delleccidio-de-la-bettola-nuovo-monumento-e-nuova-pubblicazione

Stele per i caduti di Reggio. Pdf http://www.anpireggioemilia.it/wp-content/uploads/2014/06/ReggioEmilia_ReportMonumenti_20140531.pdf

Intervista a Paolo Magnani http://gazzettadireggio.gelocal.it/reggio/cronaca/2009/02/22/news/la-mia-notte-infernale-nella-locanda-della-bettola- 1.423873 